\title{
Granulocyte-colony-stimulating factor producing esophageal squamous cell carcinoma: a report of 3 cases
}

\author{
Kojiro Eto $\cdot$ Masayuki Watanabe $\cdot$ Masaaki Iwatsuki $\cdot$ Hidetaka Sugihara $\cdot$ Asuka Murata \\ Nobuyuki Ozaki - Takatsugu Ishimoto $\cdot$ Shiro Iwagami · Yoshifumi Baba $\cdot$ Yuji Miyamoto \\ Naoya Yoshida $\cdot$ Satoshi Ikeshima $\cdot$ Masafumi Kuramoto $\cdot$ Shinya Shimada $\cdot$ Hideo Baba
}

Received: 24 September 2012/Accepted: 10 December 2012/Published online: 10 January 2013

(C) The Japan Society of Clinical Oncology 2013

\begin{abstract}
Although different types of granulocyte-colony-stimulating factor (G-CSF)-producing carcinomas have been reported, G-CSF-producing esophageal squamous cell carcinoma (SCC) is extremely rare. The prognosis of G-CSF-producing esophageal cancer has generally been reported to be very poor. Here, we report three cases of G-CSF-producing SCC who were successfully treated by use of multimodal therapy.
\end{abstract}

Keywords G-CSF-producing tumor - Squamous cell carcinoma $\cdot$ Multimodal treatment

\section{Introduction}

Different types of granulocyte-colony-stimulating factor (G-CSF)-producing carcinomas have been reported. These tumors are characterized by aggressive growth resulting in very poor prognosis [1]. Increased levels of serum G-CSF in patients with these tumors cause elevated neutrophil

K. Eto $(\bowtie) \cdot$ M. Watanabe $\cdot$ M. Iwatsuki $\cdot$ H. Sugihara .

A. Murata $\cdot$ N. Ozaki - T. Ishimoto - S. Iwagami - Y. Baba

Y. Miyamoto $\cdot$ N. Yoshida $\cdot$ H. Baba

Department of Gastroenterological Surgery, Graduate School of Medical Sciences, Kumamoto University Japan, 1-1-1 Honjo, Chuo-ku, Kumamoto 860-8556, Japan

e-mail: etouetouetou@yahoo.co.jp

H. Baba

e-mail: hdobaba@kumamoto-u.ac.jp

K. Eto $\cdot$ S. Ikeshima $\cdot$ M. Kuramoto $\cdot$ S. Shimada Department of Surgery, Yatsushiro Social Insurance General Hospital, 2-26 Matuejou, Yatsushiro,

Kumamoto 860-8556, Japan production in the bone marrow and peripheral leukocytosis. G-CSF-producing esophageal squamous cell carcinoma (ESCC) is extremely rare, and very poor prognosis has been reported. We report herein 3 cases of patients with G-CSF-producing ESCC who were successfully treated by use of multimodal treatment.

\section{Case 1}

A 59-year-old Japanese man presented with nausea, vomiting, and bloody stools. Upper gastrointestinal endoscopy revealed a tumor protruding into the lower esophagus, and the pathological diagnosis of the biopsy specimen was squamous cell carcinoma (SCC) (Fig. 1a). Computerized tomography (CT) revealed a tumor without obvious metastases located in the lower esophagus (Fig. 1b). Although the white blood cell count (WBC) was significantly elevated to $38,780 / \mu 1$ and C-reactive protein (CRP) was $0.35 \mathrm{mg} / \mathrm{dl}$, there were no other abnormal data from biochemical analysis and a coagulation study. Because a G-CSF-producing tumor was suspected, the serum G-CSF concentration was measured, and found to be $241 \mathrm{pg} / \mathrm{ml}$ (normal range $<2 \mathrm{pg} / \mathrm{ml}$ ), and the patient was diagnosed with a G-CSF-producing ESCC. He underwent esophagectomy, and the WBC and G-CSF concentration decreased to normal ranges by postoperative day 7 . Histological examination revealed moderately differentiated SCC (Fig. 1c) with a pathological stage of T3N1M0 (stage III). Seven months after surgery, the patients WBC increased to more than $20,000 / \mu \mathrm{l}$, and an increased serum G-CSF (180 pg/ml) was also observed. CT revealed a lung metastasis. He was treated by chemotherapy with docetaxel, cisplatin, and 5-fluorouracil (DCF), and remains alive 13 months after surgery. 
Fig. 1 a Upper gastrointestinal endoscopy revealed a type 1 tumor in the lower esophagus. b CT scan revealed a tumor located in the lower esophagus without definite invasion of adjacent organs and lymph nodes, or distant metastasis. c Histopathological findings of the primary tumor. The tumor was composed of moderately differentiated squamous cell carcinoma $(\times 40)$
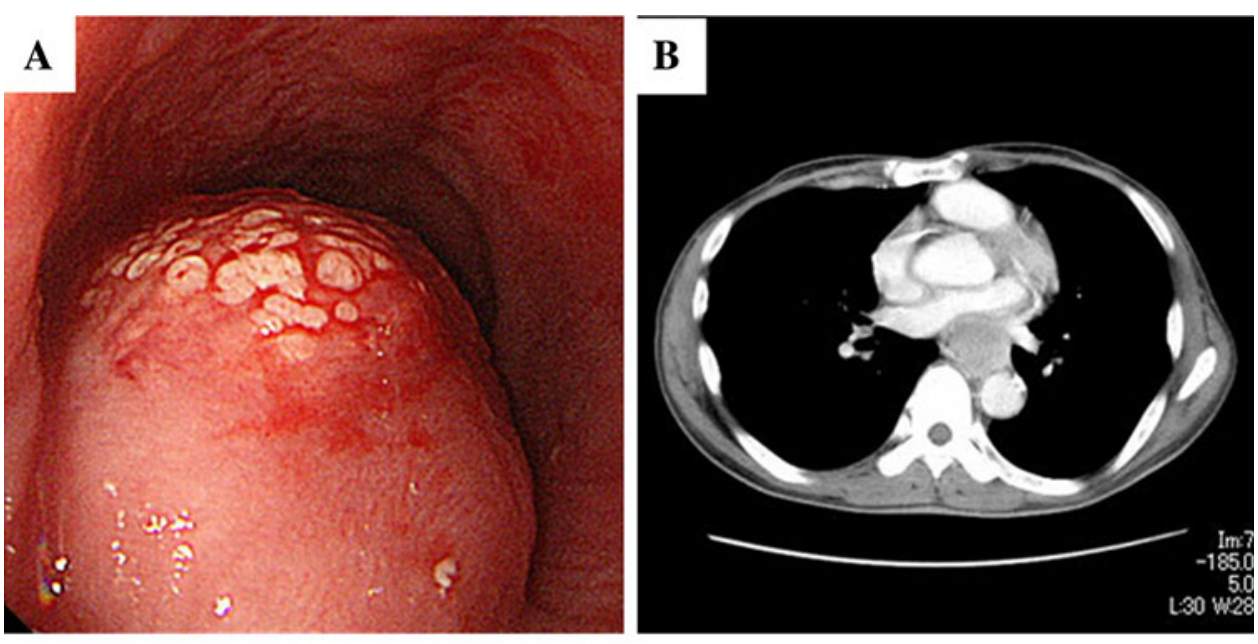

C

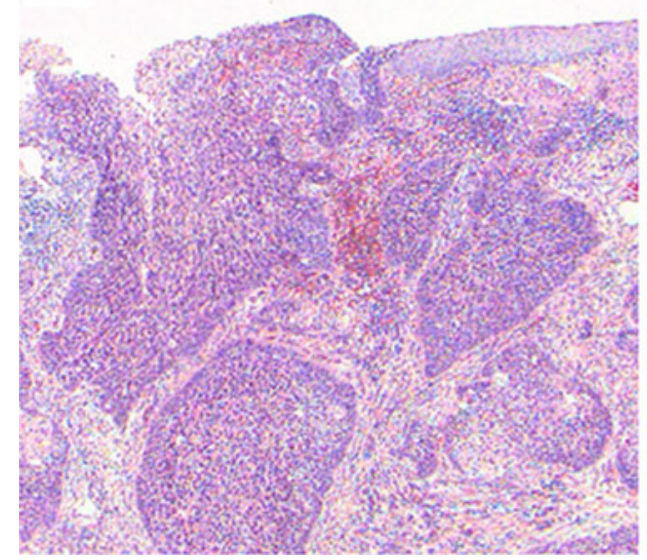

a protruding tumor at the esophagogastric junction which was diagnosed as SCC by biopsy (Fig. 3a). ${ }^{18}$ F-fluorodeoxyglucose positron emission tomography (FDG/PET) revealed a tumor located at the esophagogastric junction with multiple lymph node metastases (Fig. 3b). The WBC was $26,200 / \mu l$ and CRP was $8.44 \mathrm{mg} / \mathrm{dl}$. There were no other abnormal laboratory findings. His serum G-CSF concentration was $239 \mathrm{pg} / \mathrm{ml}$, and he was the diagnosed with a G-CSFproducing ESCC. He underwent systemic chemotherapy with the DCF regimen, for unresectable advanced ESCC with distant metastasis. After 2 courses of chemotherapy, repeat FDG/PET revealed a marked decrease in FDG accumulation both in the primary and in the metastatic lesions (Fig. 3c), and the WBC and G-CSF concentrations deceased to normal ranges. The patient is still undergoing chemotherapy and remains alive 3 months after diagnosis.

\section{Discussion}

\section{Case 3}

A 75-year-old Japanese man presented with nausea as his chief complaint. Upper gastrointestinal endoscopy revealed
G-CSF-producing carcinomas have been reported for lung, renal, gynecologic, pancreatic, hepatobiliary, gastric, and colorectal cancers [2-9]. Although G-CSF production has 
Fig. 2 a Upper gastrointestinal endoscopy revealed a type 2 tumor in the lower esophagus. b CT revealed a tumor located in the lower esophagus and right cardia lymph node metastasis without definite invasion of adjacent organs, or distant metastasis. c High accumulation was observed in both the primary lesion and the lymph node metastasis by PET. Abnormal accumulation of SUV $\max 10.8$ was observed in the primary lesion. d No viable cancer cells were observed. Histopathological examination revealed a pathological complete response $(\times 40)$
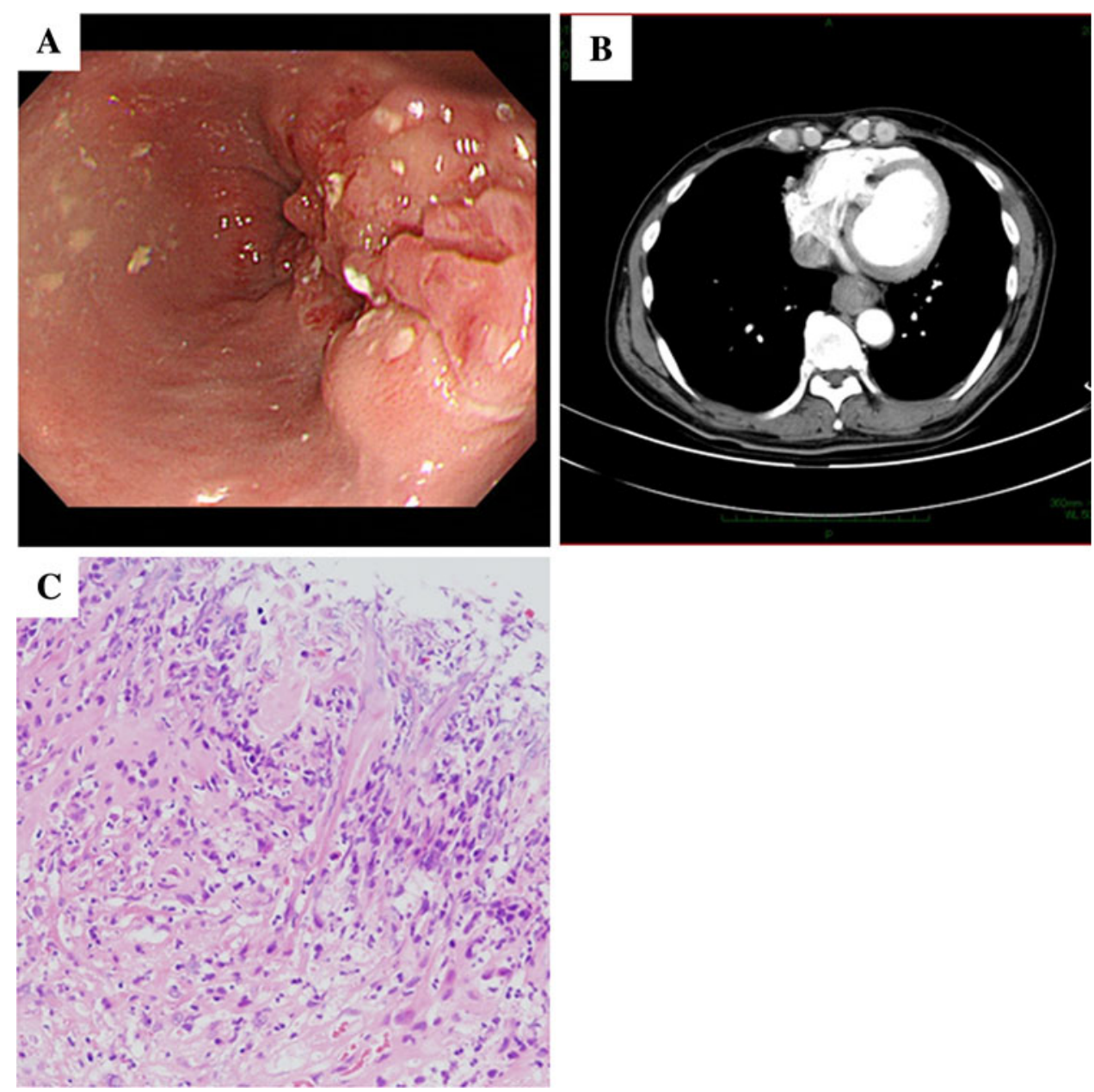

been observed, irrespective of the involved organs or histologic types, the actual frequency of G-CSF-producing tumors is unknown, because there have been no autopsy studies. Although only 4 cases of G-CSF-producing ESCCs have been reported in the literature [10-13], we treated 3 cases within a relatively short period of time, indicating that the true incidence of this type of tumor of the esophagus may be higher than previously believed.

G-CSF-producing tumors are diagnosed on the basis of four criteria:

1. extreme leukocytosis;

2. increased serum G-CSF levels;

3. improvement of leukocytosis after tumor resection; and

4. positive G-CSF immunohistochemical staining [2].

However if the first 3 criteria are satisfied, immunohistochemical staining can be omitted. In our 3 patients, both extreme leukocytosis and increased serum G-CSF concentrations were found. The leukocytosis improved after surgery in the first case and after chemotherapy in other cases. In addition, we confirmed normalization of serum G-CSF levels for all patients after their treatment.
The common features of G-CSF-producing ESCCs have previously been reported. All the affected patient were men, and all tumors were located in the lower esophagus. All tumors appeared grossly as protruding types with or without ulceration. The clinical features of our cases were similar.

G-CSF-producing tumors are believed to have high malignant potential and poor prognosis. G-CSF promotes proliferation, differentiation, and maturation of progenitor cells in the neutrophil lineage, and mobilizes hematopoietic stem cells from the bone marrow to the peripheral blood. It also activates and prolongs the survival of mature neutrophils [14]. It has been reported that G-CSF stimulates the in-vitro growth of a non-hematopoietic malignant cell line [15]. Tachibana et al. [5] reported that G-CSF production and G-CSF receptor expression by cancer cells are crucially important in mediating the malignant progression of non-haematopoietic cancer cells. It is thus possible that an autocrine growth mechanism mediated by G-CSF may lead to tumor progression. We confirmed expression of the G-CSF receptor on the cell surfaces of the specimens from our cases. This finding is indicative of 
Fig. 3 a Upper gastrointestinal endoscopy revealed a type 1 tumor from the lower esophagus to the cardiac part of the stomach. b PET revealed a tumor located in the lower esophagus, and many swelling surrounding lymph nodes. c High accumulation was observed in both the primary lesion and lymph node metastasis by PET. Abnormal accumulation of SUV max 18.4 was observed in the primary lesion

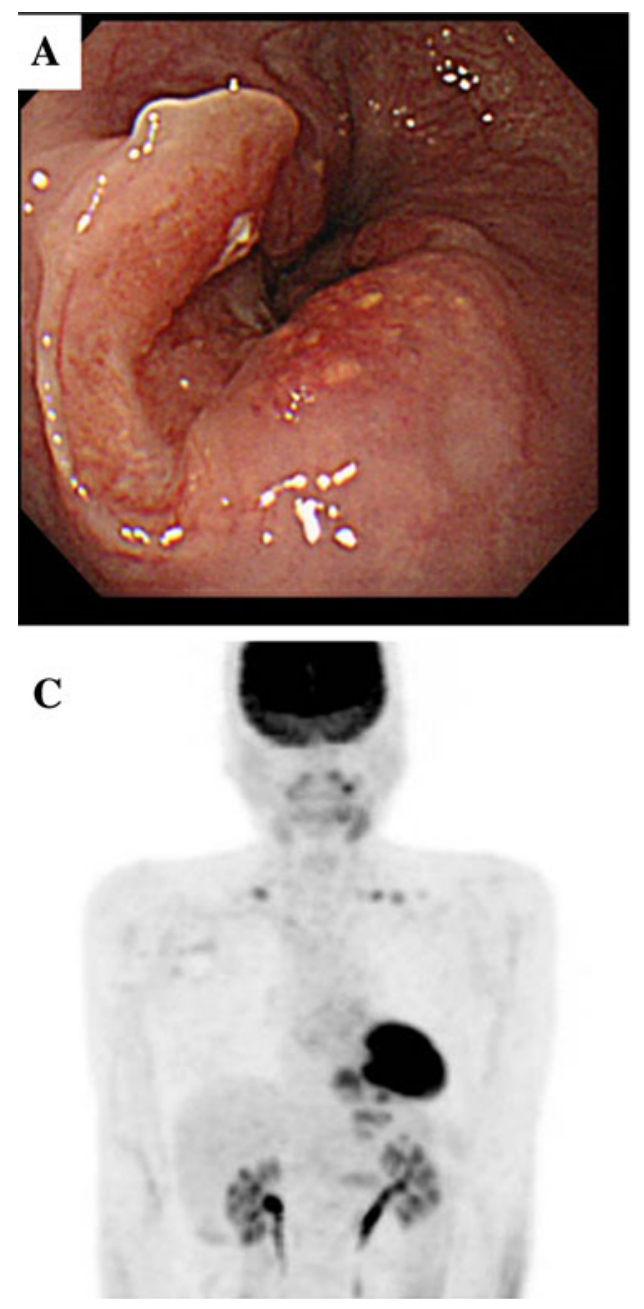

an autocrine growth mechanism mediated by G-CSF (results not shown).

Prolonged survival has been achieved for patients treated with chemotherapy or for patients treated with surgery plus neoadjuvant [16, 17] or adjuvant chemotherapy $[18,19]$. Early recurrence and poor prognosis is common for patients treated with local therapy alone, for example surgery or radiotherapy $[20,21]$. Therefore, systemic chemotherapy is the first choice of treatment for G-CSF-producing tumors, and surgery should be considered only when neoadjuvant chemotherapy has been effective. Leukocytosis can be a good marker for response or recurrence of these tumors.

We have reported 3 cases of patients with G-CSF-producing ESCC who were successfully treated by use of multimodal therapy. Although G-CSF-producing tumors are thought have a poor prognosis, it is possible that multimodal treatment may achieve long-term survival of patients with G-CSF-producing ESCC.

Conflict of interest The authors declare that they have no conflict of interest.

\section{References}

1. Kojima K, Nakashima F, Boku A et al (2002) Clinicopathological study of involvement of granulocyte colony stimulating factor and granulocyte-macrophage colony stimulating factor in nonlymphohematopoietic malignant tumors accompanied by leukocytosis. Histol Histopathol 17:1005-1016

2. Asano S, Urabe A, Okabe $T$ et al (1977) Demonstration of granulopoietic factor(s) in the plasma of nude mice transplanted with a human lung cancer and in the tumor tissue. Blood 49:845-852

3. Furihata M, Sonobe H, Iwata J et al (1996) Lung squamous cell carcinoma producing both parathyroid hormone-related peptide and granulocyte colony stimulating factor. Pathol Int 46:376-379

4. Ito T, Shimamura K, Shoji K et al (1993) Urinary bladder carcinoma producing granulocyte colony stimulating factor $(\mathrm{G}-$ CSF): a case report with immunohistochemistry. Virchows Arch A Pathol Anat Histopathol 422:487-490

5. Tachibana M, Miyakawa A, Tazaki H et al (1995) Autocrine growth of transitional cell carcinoma of the bladder induced by granulocyte-colony stimulating factor. Cancer Res 55:3438-3443

6. Baba M, Hasegawa H, Nakayabu M et al (1995) Establishment and characteristics of a gastric cancer cell line (HuGC-OOHIRA) producing high levels of G-CSF, GM-CSF, and IL-6: the presence of autocrine growth control by G-CSF. Am J Hematol 49:207-215 
7. Uematsu T, Tsuchie K, Ukai K et al (1996) Granulocyte-colony stimulating factor produced by pancreatic carcinoma. Int J Pancreatol 19:135-139

8. Takeda T, Ichiyanagi A, Sano K et al (1990) A case of gallbladder cancer producing granulocyte-colony-stimulating factor. Gastroenterol Jpn 25:762-767

9. Iwasa K, Noguchi M, Mori K et al (1995) Anaplastic thyroid carcinoma producing the granulocyte colony stimulating factor (G-CSF): report of a case. Surg Today 25:158-160

10. Matumoto G, Ise H, Kimura Y et al. (2000) Granulocyte-colony stimulating factor producing esophageal carcinoma: serum level as a marker for monitoring the effects of treatment. Int J Clin Oncol 5:328-333

11. Kato M, Osawa H, Usui $N$ et al (2002) An autopsy case of esophageal squamous cell carcinoma associated with granulocyte-colony stimulating factor production. Jikeikai Med J 49:191-195

12. Mimatsu K, Oida T, Kano H et al (2008) Aggressive progression of granulocyte colony-stimulating factor producing squamous cell carcinoma of the esophagus: case report and literature review. Esophagus 5:205-209

13. Tanabe $T$, Kanda $T$, Ishihara $N$ et al (2009) An esophageal squamous cell carcinoma patient with high serum granulocytecolony stimulating factor level: report of a case esophagus. Esophagus 6:253-258
14. Roberts AW (2005) G-CSF: a key regulator of neutrophil production, but that's not all! Growth Factors 23:33-41

15. Segawa K, Ueno Y, Kataoka T (1991) In vivo tumor growth enhancement by granulocyte colony-stimulating factor. Jpn J Cancer Res 82:440-447

16. Mori H, Shibuya T, Osada T et al (2010) Response to chemotherapy in a case of gastric adenocarcinoma producing granulocyte colony-stimulating factor. Med Sci Monit 16:CS119-CS123

17. Kyono Y, Takayama T, Kinoshita M et al (2011) Combination therapy with sorafenib and $\mathrm{S}-1$ for renal cell carcinoma producing granulocyte colony-stimulating factor. Int $\mathrm{J}$ Clin Oncol 16: 275-278

18. Takagi Y, Nakamura H, Miwa K et al (2010) A case of G-CSFproducing invasive apical cancer resected following preoperative adjuvant therapy. Thorac Cardiovasc Surg 58:304-306

19. Hasegawa S, Suda T, Negi K et al (2007) Lung large cell carcinoma producing granulocyte-colony-stimulating factor. Ann Thorac Surg 83:308-310

20. Kawaguchi M, Asada Y, Terada T et al (2010) Aggressive recurrence of gastric cancer as a granulocyte-colony-stimulating factor-producing tumor. Int J Clin Oncol 15:191-195

21. Matsumoto Y, Mabuchi S, Muraji M et al (2010) Squamous cell carcinoma of the uterine cervix producing granulocyte colonystimulating factor: a report of 4 cases and a review of the literature. Int J Gynecol Cancer 20:417-421 